\title{
Precariedad, Precariado y Precarización. Un comentario crítico desde América Latina a The Precariat. The New Dangerous Class de Guy Standing
}

\author{
Hernán Cuevas Valenzuela \\ Universidad Diego Portales, Santiago de Chile. \\ Email: hcuevasster@gmail.com
}

\begin{abstract}
Resumen: Este ensayo revisa críticamente uno de los libros de sociología del trabajo más influyentes de los últimos años: The Precariat. The New Dangerous Class, de Guy Standing. Se describen los principales argumentos del libro en el contexto de la discusión sobre la formación y expansión del capitalismo, la formación de las clases sociales trabajadoras, y la ciudadanía. A partir de la lectura de The Precariat, este ensayo distingue los diferentes mecanismos generalizados que producen la precariedad laboral asociados a los procesos de globalización, inseguridad social y flexibilidad laboral, y propone que es necesario avanzar en la comprensión de los modos en que dichos mecanismos se articulan entre sí y en que se acoplan (o desacoplan) con los contextos sociales e históricos más amplios de cada realidad. En relación a lo anterior, se discute la expansión del capitalismo, la formación de clase y la cuestión de la ciudadanía. Finalmente se concluye que las reflexiones de Standing son útiles para pensar la situación del trabajo en América Latina.
\end{abstract}

Palabras clave: precariado, precarización, inseguridad, capitalismo, trabajo flexible, globalización

\section{Precariety, Precariat and Precariousness. A critical comment from Latin America to The Precariat. The New Dangerous Class by Guy Standing}

\begin{abstract}
This paper critically reviews one of the most influential books in recent years in the field of sociology of work: The Precariat. The New Dangerous Class by Guy Standing. The essay describes the main arguments of the book within their wider intellectual and historical contexts. Based on The Precariat, this essay distinguishes between different generalized mechanisms of production of precarious labour associated to globalization, labour flexibilization and social insecurity. The essay moves further The Precariat, in proposing the need to consider the articulation between these mechanisms and their adjustment (or lack of it) to the wider social and historical context of each reality. The essay also discusses the formation and expansion of capitalism, the formation of the working classes, and citizenship. The paper concludes that the Standing analysis is useful to think the work situation in Latin America. globalization

Keywords: precariat, precarization, insecurity, capitalism, flexible work,
\end{abstract}




\section{Precariedade, precariado e precarização. Um comentário crítico desde América Latina para The Precariat. The New Dangerous Class de Guy Standing}

Resumo: Este ensaio analisa criticamente um dos livros mais influentes da sociologia do trabalho nos últimos anos: The Precariat. The New Dangerous Class de Guy Standing. Descrevem-se os principais argumentos do livro no contexto da discussão sobre a formação e expansão do capitalismo, a formação das classes sociais trabalhadoras e a cidadania. A partir da leitura do $\mathrm{O}$ precariado, este artigo distingue os diferentes mecanismos causadores da precariedade laboral associada com os processos de globalização, insegurança social e flexibilidade do trabalho, e propõe que é necessário avançar na compreensão das formas pelas quais estes mecanismos estão interligados entre eles e como se superpoem (ou dissociam) com os contextos sociais e históricos mais amplos de cada realidade. Em relação a isto, discute-se a expansão do capitalismo, a formação das classes e a questão da cidadania. Por fim, conclui-se que as reflexões de Standing são úteis para pensar a situação do emprego na América Latina.

Palavras-chave: precariado, precariedade, insegurança, capitalismo, trabalho flexível, globalização

$* * *$

\section{Introducción}

Si bien se ha hablado de precariedad hace bastante tiempo (por ejemplo, Day, 1952), los términos trabajo precario, precarización, precariado y, por supuesto, precariedad, han adquirido mayor notoriedad desde los 2000. Y esto, tanto en la práctica política de activistas como en el discurso académico. Ahora bien, mientras el movimiento EuroMayDay (2004) puso el concepto de precariedad en la palestra política, haciendo más evidente la precariedad a nivel internacional, por su parte, el mundo académico ha tratado algo tardíamente esta postulada nueva realidad.

Entre los esfuerzos académicos de comprensión de la precariedad laboral destaca el trabajo de Guy Standing, conocido intelectual público que ha sido profesor de la Universidad de Bath, Investigador de la OIT y que actualmente se desempeña en SOAS (School of Oriental and African Studies) de la Universidad de Londres. Standing ha escrito un brillante ensayo acerca de la precariedad: The Precariat. The New Dangerous Class (2011) (en adelante, The Precariat). En las próximas páginas me propongo presentar algunos de sus argumentos y desarrollar unas breves notas acerca de su utilidad para comprender la situación en América Latina. Pero antes de desarrollar estos contenidos, parece pertinente a modo de contexto señalar algo más acerca de Guy Standing. Además de un reputado especialista en asuntos laborales, Standing es un conocido defensor de las políticas de ingreso básico universal garantizado para cada ciudadano sin mediar exigencia alguna ni trabajo. En ese plano, se ha desempeñado como copresidente de BIEN (Basic Income Earth Network). Durante su destacada carrera académica, Standing ha sido un implacable crítico de los excesos de 
la mercantilización de la sociedad. Gran Bretaña, país donde se ha desempeñado principalmente el autor, es sin duda un caso ejemplar de mercantilización de la sociedad. Los enormes costos sociales de este proceso han quedado retratados no sólo en las investigaciones de cientistas sociales británicos como Stuart Hall (2011), Stuart Hall y Alan O’Shea (2013) o David Harvey (2007), sino que también en el cine social británico de las últimas décadas por medio de filmes como Brassed Off (1996) de Mark Herman, The Full Monty (1997) de Peter Cattaneo y, especialmente, My Name is Joe (1998), Navigators (2001) y It's a Free World (2007) de Ken Loach. Todos estos filmes muestran de un modo equivalente a como lo hizo en el siglo XIX la así llamada social problem novel de autores como Charles Dickens, cómo los británicos vivieron de manera temprana la crisis del fordismo y la desindustrialización, el fin del proyecto de la socialdemocracia de postguerra, el subsiguiente apogeo del capitalismo financiero y la instalación del neoliberalismo en sus versiones de derecha (Margaret Thatcher) y progresista (Anthony Blair). El caso británico, sin duda alguna, provee mucho material para un libro como The Precariat. Sin embargo, el libro abunda en referencias a los Estados Unidos de Norteamérica, Australia, Japón, Alemania, España, Italia, China e India, para nombrar sólo algunos casos con los que Standing ilustra sus argumentos. América Latina está casi ausente y, en general, el Sur Global es tratado de manera secundaria. A pesar de ello, hacia el final de estas breves páginas sostendré que la reflexión de Standing es valiosa para pensar la actual situación del trabajo en América Latina. En lo inmediato nos concentrarnos en la exposición de los argumentos del autor.

¿Qué son la precarización, el precariado y la precariedad? En The Precariat. The New Dangerous Class Standing define al precariado como una clase social en formación (volveremos sobre esto) y a la precarización como un proceso en que el sujeto es sometido a presiones y experiencias que lo conducen a vivir una existencia frágil en el presente, sometido a incertidumbres acerca del futuro, con una identidad insegura y carente de un sentido de desarrollo posible por medio del trabajo y el estilo de vida (2011:16-18). The Precariat es a la vez la continuación y un resumen de la investigación previa de Standing acerca de la precarización del trabajo y la formación del precariado que se hallaba previamente delineada en algunos de los capítulos de Work After Globalization. Building Occupational Citizenship (2009). Conjuntamente, estos trabajos ofrecen respuestas interesantes -y a la vez polémicas- a la pregunta por la precariedad.

El objetivo de Guy Standing en The Precariat es dar cuenta de los efectos sociales y políticos de la precarización del trabajo y su posible remedio. El libro está escrito en un lenguaje a la vez claro y accesible para el lego, con un estilo ágil que evita detener al lector con tediosas series de datos y debates bizantinos de interés únicamente académico. El libro está organizado en 7 capítulos de dimensiones amables, en el que el autor logra resumir años de investigación acerca de la evolución del trabajo y la formación del la clase social que llama precariado. Todo esto podría llevar a pensar que The Precariat es una obra ensayística de divulgación. Pero, 
como lo evidencian múltiples revistas especializadas, su impacto en el mundo académico ha sido amplio y la discusión que ha generado valiosa. ${ }^{1}$ En definitiva, su objetivo a la vez analítico, polémico y propositivo, hacen de The Precariat un libro de difícil clasificación, pero a la vez imprescindible y de gran interés para los científicos sociales.

Uno de los argumentos centrales que subyacen al relato de The Precariat es que la lógica de la mercantilización -o comodificación preferirían algunos- ha llevado a la formación no ya de economías de mercado, sino de verdaderas sociedades de mercado. ${ }^{2}$ Standing reprocha la comodificación de la vida en general y, en especial, la re-comodificación del trabajo por medio de la liberalización del mercado laboral bajo la influencia de políticas neoliberales. Vale la pena detenernos por un momento en esta tesis de la re-comodificación del trabajo y la mercantilización de la sociedad y sus antecedentes.

Esta no es una idea del todo novedosa, pues tiene su antecedente en La Gran Transformación de Karl Polanyi (1992, original de 1944) y, por supuesto, en El Capital (volumen I) de Karl Marx (2001). La comodificación es aquel proceso característico y a la vez fundamental de la economía capitalista que transforma los objetos, bienes, o incluso ideas y objetos usualmente no considerados bienes transables, en mercancías. A diferencia de Marx, cuyo punto de mayor interés fue la acumulación capitalista vía extracción del plus valor (explotación), para Polanyi el nudo gordinao del capitalismo -el verdadero asunto a comprender- es la lógica de la comodificación capitalista y la institución social del mercado. La expresión La Gran Transformación alude a la conversión de los factores productivos -trabajo humano, tierra, naturaleza- en mercancías intercambiables en el mercado. Contra quienes como Adam Smith definieron al mercado como una organización natural del intercambio, Polanyi plateó que el mercado es una institución social, que además tiene por supuesto una concepción antropológica individualista, atomista y egoísta de la condición humana, la que tampoco es natural. En efecto, Polanyi demostró que las sociedades capitalistas crean "mercados auto-regulados" capaces de comodificar incluso la tierra, el trabajo y la moneda, que no son bienes (o servicios) ordinarios, transformándolos de este modo en “mercancías ficticias” (Polanyi, 1992: 68-76). Las palabras con que denominamos sus precios -renta, salario e interés- reflejan hasta cierto punto su singularidad en tanto mercancías ficticias.

Pero esta es sólo una parte de la historia de La Gran Transformación. Polanyi desarrolló una teoría del desarrollo capitalista caracterizado por un movimiento pendular de fases de comodificación y decomodificación. Este doble proceso juega un rol fundamental tanto en la formación y expansión del capitalismo, como en su estabilización vía regulación. El término decomodificación -el otro movimiento del péndulo- se refiere al proceso por medio del cual un objeto ya mercantilizado es sacado del mercado. Este es el movimiento contrario al laissez faire de los mercados auto-regulados que producen altos costos sociales. Por ejemplo, el estado de bienestar y los regímenes de negociación colectiva centralizados, que 
fueron característicos de varias de las economías más avanzadas de Europa, favorecieron la decomodificación del trabajo por medio de varios mecanismos, entre los que destacaron las políticas sociales y servicios públicos que otorgaban seguridad a los trabajadores y sus dependientes, la regulación política del precio del trabajo, y las políticas de pleno empleo.

El foco de atención de Guy Standing en The Precariat es la recomodificación del trabajo (2011: 41-42). El término recomodificación describe los más recientes procesos de reducción de los beneficios sociales otorgados por las empresas y las políticas sociales de bienestar, el repliegue del estado y sus servicios, la limitación de la negociación colectiva, y el incremento de la desregulación y flexibilización del mercado laboral. El efecto de este conjunto de procesos ha sido una renovada mercantilización del trabajo, que con la importante contribución e influencia de la ideología neoliberal, ha profundizado las condiciones de precarización social (Standing, 2009; 2011).

\section{La Precariedad, los mecanismos de precarización y la aparición del precariado}

El término precario deriva del latín precarius, que se refiere a aquello que se obtiene por medio de la petición, la súplica y el ruego. En el derecho romano, precarium era un tipo de contrato en que el beneficiario arrendaba un bien que podía ser reclamado de vuelta por el dueño en cualquier momento. Se trataba, por lo tanto, de un arriendo relativamente inseguro, pues el arrendador estaba sometido en todo momento al riesgo del reclamo del bien por parte del propietario.

Como vemos, la etimología del sustantivo precariedad y del adjetivo precario es reveladora, pues asocia estos términos a la experiencia de la pobreza, a la carencia de recursos propios, a la dependencia de quien carece respecto de quien posee bienes, a la falta de autosuficiencia, y a la inseguridad de quien debe pedir, suplicar, o solicitar favores para autosustentarse.

Por su parte, el término precariado es una conjunción de las palabras precario y proletariado (Standing, 2011: 7). Este neologismo alude a un estrato o segmento social particular que ha aparecido en sociedades capitalistas de regímenes laborales crecientemente desregulados y de trabajo flexible. ${ }^{3}$ En The Precariat, Standing sostiene que el crecimiento del precariado es el resultado de dos procesos revolucionarios: la globalización y el neoliberalismo. Estas revoluciones, que han afectado a buena parte del mundo desde al menos los inicios de la década de 1980, han promovido la competencia global a niveles sin precedentes. La competencia global ha tenido varios efectos. Entre ellos, Standing destaca los siguientes:

(1) el debilitamiento del poder del factor trabajo

(2) la adopción de numerosas formas de trabajo flexible por parte de 
las empresas que son expuestas a la necesidad de ajustarse a las competencia global con economías con bajos costos laborales (especialmente China e India), y

(3) la susceptibilidad de los estados a la introducción de políticas de desprotección social y desregulación y flexibilización del mercado laboral.

Otra de las tesis fundamentales de The Precariat, la que es compartida con otros estudios sobre la precariedad laboral (por ejemplo, Castel, 2009 y Dörre, 2009;) es que este proceso generalizado de retroceso del así llamado estado de bienestar explicaría la vuelta de la inseguridad social en varias economías avanzadas de Europa (Standing, 2011; 2012). Volveremos a este punto hacia el final para evaluar su importancia para la delimitación del concepto de precarización y su efecto sobre la aplicabilidad del mismo al análisis del Sur Global y de América Latina en particular, pues éste parece ser el factor más problemático de dicha conceptualización. En lo inmediato, expondré cuáles son los mecanismos productores de precariedad según Standing, para lo cual propongo ordenarlos en tres grupos de mecanismos asociados, a saber: (a) los procesos de flexibilización del mercado laboral, (b) el aumento de la inseguridad social y (c) los efectos de la globalización sobre la economía.

La flexibilidad del mercado del trabajo es el principal mecanismo de precarización laboral, y su funcionamiento tiene varias dimensiones que son necesarias de considerar:

(1) La flexibilidad salarial, referida a la posibilidad de ajustar el precio de la mano de obra según los cambios de la demanda de trabajo (Standing, 2011: 40).

(2) La flexibilidad de número, la que se refiere al empleo a la capacidad de contratar o despedir trabajadores sin mayores costos para las empresas (Íbid: 31-36).

(3) La flexibilidad funcional, que se refiere a la discrecionalidad de la dirección de las empresas para organizar el trabajo, cambiar la estructura de la empresa y las funciones de los trabajadores (Íbid: 3640).

(4) En cuarto lugar está la flexibilidad entendida como la capacidad de la dirección de las empresas para definir con relativa soberanía las competencias y habilidades requeridas por sus trabajadores, con lo cual la empresa (y no tanto el trabajador) se convierte en el foco de las decisiones referidas a formación y capacitación laboral (Íbid: 6)

A estas formas de flexibilidad, Standing agrega otras causas de la precarización asociadas a la inseguridad social, como son: 
(5) La inseguridad causada a nivel macro en el mercado del trabajo debido a la carencia o debilidad de políticas de pleno empleo, que afectan las oportunidades de obtención de un salario suficiente.

(6) La inseguridad causada por la fragilidad de los sindicatos y/o las restricciones al derecho de huelga que disminuyen la capacidad de representación y negociación colectiva de los trabajadores en el mercado del trabajo.

(7) La inseguridad producida por la ausencia o debilidad de la protección contra accidentes de trabajo y enfermedad, o por las insuficiencias de las regulaciones de la seguridad, higiene y condiciones de salud en el lugar de trabajo.

(8) La inseguridad provocada por la falta o insuficiencias de regulaciones, protecciones, y garantías estatales referidas a los derechos socioeconómicos de las personas. La ausencia de sueldo mínimo o seguro de desempleo, la mala provisión de servicios sociales básicos como salud y educación, y la insuficiencia de políticas redistributivas.

Junto con las flexibilización del trabajo (1-4) y las formas de precarización de la seguridad social (5-8), es necesario considerar a la competencia global (9), la globalización de los mercados (10) y la flexibilidad con que se movilizan los capitales que prefieren economías de bajos costos laborales(11). Estos tres últimos factores, asociados a la constitución de una economía capitalista de escala global, han contribuido a la producción de una mayor precarización del trabajo, incluso en varias economías avanzadas. Economías hasta hoy exitosas, como Alemania, han debido bajar sus costos de empleo y prácticamente congelar los salarios para mantener su competitividad (Dörre, 2009). Arne Kalleberg (2012a; 2012b) explica que la precarización y formación de trabajos de poca calidad ha sido una respuesta empresarial a la intensificación de la competencia global de precios, lo que se suma a la declinación de la intervención del estado en el mercado laboral y el debilitamiento del poder de los sindicatos.

Standing está en lo correcto al destacar estos factores como mecanismos productores de precariedad -aunque él no use esa terminología-. Lo que no detalla suficientemente es la naturaleza de cada uno de dichos factores, ni la clase de relación que tienen entre sí, ni con sus contextos más amplios. Pero no es justo criticar a un autor por la tarea que correspondería tal vez a una disciplina completa (¿la sociología del trabajo? ¿la economía política?).

Concuerdo en que estos sí son los principales mecanismos generalizados de producción de la precariedad laboral en el capitalismo de la modernidad tardía. Sus efectos se dejan sentir en todos los confines del sistema capitalista mundial. Sin embargo, esto ocurre de manera diferente en cada sociedad. En otras palabras, los diferentes contextos territoriales, so- 
ciales, económicos, políticos, institucionales y culturales en que se desarrolla la relación entre capital y trabajo -que es necesariamente una relación territorializada, esto es, localizada y globalizada a la vez- y sus respectivas historicidades, obligan a considerar las particulares tonalidades que, en cada caso, adquieren tanto los mecanismos productores de la precariedad, como también la precariedad laboral misma. Por ejemplo, la particular deriva histórica europeo continental que llevó al desarrollo de regímenes de seguridad social, y sus diferencias respecto de las economías liberales de Gran Bretaña y los Estados Unidos de Norteamérica, o de distintas historias nacionales en América Latina, constituye mucho más que un mero telón de fondo para el desarrollo de la precariedad en las economías de Europa continental. La formación que adquiere el precariado, e incluso si ocurre su formación (o no) como clase, y la modalidad que adquiere la precariedad laboral (y su profundidad), está condicionada en parte importante por este contexto complejo, en el que funcionan estos mecanismos. Por ello, es un error considerar estos mecanismos como si produjeren sus efectos de precariedad en un vacío histórico y de manera homogénea. En este punto, sostengo que los conceptos elaborados por Standing pecan de cierta ahistoricidad y requieren de ser pensados en contextos específicos con mayor detalle.

Si observamos con atención, también en el campo de los efectos de la precarización apreciaremos una heterogeneidad de resultados que, lejos de ser aleatorios, responden a los acoples concretos que se da entre los mecanismos generales de producción de la precariedad con los contextos particulares. De este modo, acá también será necesario comprender la dialéctica entre lógicas generales de carácter global, y la historicidad de los contextos en que dichos efectos acaecen. Por supuesto, esto requiere del desarrollo de una agenda de investigación que sin duda desborda los objetivos de este breve escrito.

En lo que queda, nos concentraremos en tres clases de efecto de los mecanismos que producen la precarización del trabajo: la compresión y aceleración del tiempo de trabajo, la fragmentación de la estructura de clases, y la constitución de denizens (o semi-ciudadanos).

En The Precariat, Standing (2011: 115-131) sostiene que la flexibilización del trabajo promueve una creciente adaptación de la vida humana a los ritmos del capitalismo que ha llevado a la compresión del tiempo y ha dañado la experiencia individual y comunitaria y disminuído el control de las personas sobre la propia vida. Este no es un tema nuevo. Por ejemplo, hace más de medio siglo Edward Palmer Thompson relató en Time, Work-Discipline and Industrial Capitalism (1967) y en parte en La Formación de la Clase Obrera Inglesa (1989, original de 1963) cómo sucedió históricamente la adaptación de la experiencia de los trabajadores ingleses a los ritmos de la producción industrial capitalista y al tiempo cronológico. Se produjo así un definitivo alejamiento de las formas tradicionales de medir el paso del tiempo, ancladas en la agricultura y las estaciones. Esta adaptación fue brutal, y sometió a generaciones de trabajadores y sus familias al 
ritmo de vida inhumano. Las luchas del siglo XIX para fijar una jornada de trabajo, que parecen hoy cosa del pasado, fueron fundamentales y ayudaron a la humanización del trabajo al fijar una jornada laboral razonable. Pero, como lo sugiere Standing, estas luchas por la regulación del tiempo de la jornada de trabajo adquieren nueva relevancia hoy. En nuestros días, el trabajo somete incluso el pulso biológico del cuerpo a ritmos cada vez más acelerados. El trabajo humano debe adaptarse ahora a los ritmos de las tecnologías de comunicación e informática y al trabajo en redes y cadenas de valor internacionales que son en verdad globales y que no cesan su actividad. Para muchos trabajadores precarizados que conforman fuerzas de venta, equipos de publicidad y relaciones públicas, diseñadores, informáticos, académicos e investigadores, y una miríada de trabajadores por cuenta propia, hoy su trabajo se realiza en cualquier momento, pues su actividad ya no está gobernada por una jornada de trabajo reglada, sino que por los requerimientos de la empresa, del cliente, por metas individuales y de equipo, por proyectos, y por una estructuras de incentivos económicos al desempeño. Por supuesto, nada de esto obedece las limitaciones de la jornada laboral de 8 horas. Más aún, el tiempo de trabajo ha invadido la intimidad y colonizado el tiempo libre. Algo similar ocurre con el lugar de trabajo, que se difunde y difumina, abarcando en ocasiones espacios familiares. Así, las fronteras entre el tiempo libre y el tiempo de trabajo, el lugar de trabajo y el hogar, se vuelven porosas o incluso desaparecen. Estos procesos comprimen la experiencia temporo espacial, lo que contribuye a que, finalmente, los trabajadores precarizados tengan menos control sobre su tiempo. Este proceso es verificable globalmente. Sin embargo, adquiere tonalidades propias en cada contexto. En América Latina, por ejemplo, la larga historia de formas de desprotección laboral y trabajo informal, en cierto modo facilitan la introducción de regímenes actuales de trabajo precario, como son las variadas formas de trabajo eventual (temporal, por obra o faena, etc.), trabajo por tiempo parcial, sub-contrato, o incluso formas de teletrabajo.

Otro efecto del proceso de precarización del trabajo es la fragmentación de la estructura de clases ocupacionales y su reconfiguración. Standing (2011, 2012) plantea que la nueva estructura de clases estaría constituida por siete grupos: 1) una elite económica de individuos ricos, 2) el salariat, constituido por trabajadores privilegiados por el sistema, asalariados que mantienen trabajos formales estables de tiempo completo e ingresos altos, 3) los proficians (professionals y technicians) que son profesionales y técnicos sin seguridad de empleo -y en ese sentido son trabajadores precarizados- pero que dado el conocimiento experto que poseen pueden obtener altos ingresos, 4) el tradicional proletariado que constituyó el corazón de la clase trabajadora en los países que vivieron el desarrollo industrial y que aún gozan de algunos beneficios sociales y niveles de seguridad laboral relativamente más altos que otras clases ocupacionales, 5) el precariado (precariat, de precario - precarious en inglés- y proletariado -proletariat en inglés-.), constituido por trabajadores que se desempeñan en trabajos altamente inseguros, 6) los desempleados que constituyen un "ejército de reserva" de mano de obra barata y 7) el lumpenproletariado 
conformado por los marginalizados de la sociedad (drogadictos, enfermos mentales, criminales e individuos atrapados en una situación de anomia crónica) (Standing 2011: 7-13 y 2012: 589).

El precariado, según Standing (2011), es la clase más característica de capitalismo financiero. Se trata de una clase en formación, sin una memoria colectiva común, carente del tipo de identidad que caracterizó al proletariado industrial y, en consecuencia, con una solidaridad frágil. Al respecto, la imagen escogida para la portada del libro es sugerente: un grupo de operarios anónimos, deliberadamente representados como cuerpos sin rostro.

El precariado es internamente muy variado y heterogéneo. Incluye a migrantes, mujeres, jóvenes, discapacitados, individuos criminalizados, trabajadores maduros dados tempranamente de baja, y trabajadores mayores con pensiones insuficientes que los obligan a volver al mercado laboral en condiciones de mayor inseguridad. Otro signo de su heterogeneidad es que incluye tanto a personas con baja calificación como también a trabajadores altamente calificados.

El precariado no sólo está internamente muy fragmentado en diversas posiciones y ocupaciones objetivas, como hemos visto. Además se haya diferenciado en posiciones subjetivas distintas. En efecto, el precariado consiste tanto de sujetos sonrientes (grinners) que escogen voluntariamente el trabajo temporal y por tiempo parcial, como de sujetos que se quejan (groaners) que no pueden sino optar por trabajos precarios (Íbid: 59). Aunque parecen muy distintos, según Standing (Íbid: 19-24) tienen en común la experiencias de:

(1) Rabia y resentimiento debido a que sus aspiraciones se han visto frustradas.

(2) Anomia entendida como una forma de desesperanza aprendida debido a que no encuentran un trabajo significativo y satisfactorio.

(3) Ansiedad debido a su permanente exposición a las inseguridad laboral, y

(4) Alienación debido a que frecuentemente experimentan falta de motivación y carecen de respeto social.

Standing sugiere que éstas emociones son comunes a los grinners y groaners, pero no es del todo claro cómo subyacen ellas a dos clases de relatos y autocomprensiones tan diferentes. Como ha señalado Richard Sennett hace más de dos décadas en La Corrosión del Carácter (2000), las condiciones y efectos sobre las personas del capitalismo flexible se reflejan en los aspectos subjetivos de la experiencia de precariedad (aunque Sennett no use este término), y aunque en el habla de algunos trabajadores éstos expresen su satisfacción con la autonomía lograda en el nuevo régimen laboral, Sennett detecta por medio de entrevistas y conversaciones infor- 
males que incluso estos trabajadores aparentemente "beneficiados" por el nuevo régimen laboral, más profundamente también resienten la inseguridad, desorientación y corrosión del sentido de identidad. Esta experiencia individual y colectiva de la precariedad puede ser concebida como una especia de síndrome de la precariedad.

Una de las tesis más discutibles de Standing es la que sostiene que las características del precariado -especialmente sus emociones subjetivas- lo vuelven una clase peligrosa para la estabilidad de las democracias. En efecto, Standing sostiene que estas características objetivas y subjetivas hacen del precariado una masa susceptible a los llamados de sirenas de movimientos extremistas y neo-fascistas. A esto agrega Standing que la heterogeneidad del precariado hace difícil su organización política según el modelo del proletariado industrial y la sindicalización obrera. Recientemente Standing (2012) ha planteado que los grupos dispares que constituyen el precariado están siendo amalgamados bajo una común protesta y demanda contra la ola de programas de ajuste y medidas de austeridad en Europa.

En el caso de América Latina, la heterogeneidad de la estructura social tiene sus propias singularidades, con lo que el precariado en tanto clase en formación parece hallarse aún menos delineada que lo señalado por Standing para las economías del Norte Desarrollado. Además de la división de clase (y secundariamente de edad, género y nivel de calificación), en América Latina la división de raza o etnia, e incluso la división rural/urbano, son en muchas sociedades factores que complejizan aún más la formación social de clase, agregando otras intersecciones conflictivas que demandan su consideración. Por su parte, los factores subjetivos de la formación de clase del precariado deben ser reconsiderados en vista de factores como la experiencia de desesperanza aprendida, de la amplitud de la pobreza dura, de la profundidad de las desigualdades sociales y la pervivencia de formas de opresión, marginación y exclusión de larga historia en la región.

Otra característica del precariado descrita por Standing (2011) es que éste estaría conformado en parte importante por denizens, es decir, por sujetos que no gozan de todas las garantías que provee el status de ciudadano. ${ }^{4}$ Tal es el caso de los asilados, trabajadores indocumentados, trabajadores extranjeros con permiso de trabajo temporal, y residentes legales con derecho a trabajar indefinidamente. A pesar de contribuir en varios aspectos a las comunidades nacionales en que habitan, incluido el pago de impuestos, frecuentemente carecen de algunos beneficios sociales y, especialmente, de derechos políticos. También otros grupos sociales constituidos por 'nacionales' caen frecuentemente en la categoría de denizen. Estas son las minorías culturales, raciales, minorías sexuales, y las mujeres.

Uno de los mayores aciertos de The Precariat es la consideración crítica de la relación entre ciudadanía y trabajo, que ha sido fundamental en la conformación de la ciudadanía moderna bajo la figura ciudadano-trabajador. Este es un tema olvidado o inexplicablemente dejado de lado, y cuya 
relevancia es hoy más evidente en un mundo globalizado con una creciente movilidad del trabajo. Los regímenes laboral y de ciudadanía tienen efectos ambivalentes, pues no sólo incluyen por la vía de formas de reconocimiento, sino que también excluyen o, en el mejor de los casos, incluyen de manera diferenciada o parcial a amplios grupos sociales. La ciudadanía y los regímenes laborales incluso sirven al propósito de construir líneas de opresión, explotación, discriminación y exclusión (Cuevas, 2012). En América Latina la larga historia de formas de reconocimiento y des-conocimiento (en el sentido de misrecognition) y de formas de trabajo más o menos forzoso como la esclavitud, la encomienda, la repartición de indios, y las formas más recientes del inquilinaje, una serie de modalidades clientelares de contratación, la variedad de esquemas de trabajo informal, etc., otorgan a la experiencia latinoamericana de denizen un trasfondo histórico específico. De este modo, la experiencia contemporánea de semi-ciudadanía de trabajadores Latinoamericanos en y fuera de América Latina debe ser comprendida en contexto del complejo de sus historicidades, que incluyen desde antaño formas de semi-ciudadanía y precariedad. A ello hay que agregar tanto el exiguo desarrollo de los derechos sociales en la región, como la conculcación de derechos civiles y políticos bajo regímenes de fuerza hasta tiempos recientes. Estos elementos históricos, en lugar de volver inútil la distinción entre citizen-denizen, le otorgan a la discusión sobre la relación entre ciudadanía y trabajo una actualidad y urgencia incluso mayor que la que tiene en otras latitudes. Las luchas de los trabajadores precarios en América Latina, como sucede por ejemplo con los trabajadores inmigrantes y/o indocumentados, frecuentemente se inscribe en una historia de persecución y racismo naturalizado, como se aprecia en el excelente film argentino Bolivia (2001) dirigido por Israel Adrián Caetano. También el clasismo es un patrón cultural normalizado, como se observa en el film chileno La Nana (2009) de Sebastián Silva. Las formas particulares del clasismo y el racismo en América Latina agregan intersecciones críticas con tintes singulares a la experiencia de ciudadano-trabajador.

\section{A modo de conclusión}

Hemos planteado con un tono de escepticismo que la tesis de Standing (2011) de la formación del precariado a partir del retroceso del estado de bienestar y la flexibilización y desregulación del trabajo tiene que ser revisada para tener alguna aplicabilidad en sociedades que, como es el caso de América Latina, nunca vieron la formación de un estado de bienestar en forma. Desde este punto de vista, lo que Standing denomina precarización aparece como lo corriente, como el estado normal del trabajo, y la experiencia de protección y seguridad social como la excepción y casi como un privilegio de clases medias y acomodadas.

En efecto, es posible sostener que en las sociedades del así llamado Sur Global -que incluye a África, Asia, y América Latina- la precariedad laboral más que una excepción o una realidad emergente en tiempos recientes, parece haber sido una característica constante del desarrollo desigual y 
combinado en el capitalismo y que afecta a buena parte de las categorías ocupacionales del heterogéneo mercado laboral (Webster, 2005). Ahora bien, la manera tradicional de concebir la condición laboral histórica de lo trabajadores en América Latina por medio de la categoría de informalidad laboral también se ha vuelto problemática (Leiva, 2012). En efecto, si bien la categoría de informalidad sigue ofreciendo una mirada valiosa para explicar en parte importante la heterogeneidad de los mercados del trabajo y las inequidades sociales que crónicamente se incubaron en América Latina (Sproll, 2013), hoy ésta categoría parece insuficiente. Por ejemplo, hoy encontramos que en sectores productivos como la agroindustria y el sector portuario en Chile las relaciones laborales se han formalizado y no por ello son más seguras y estables. Las variedad de formas de contrato permitidos y la diversidad de relaciones laborales existentes en el campo -en especial el aumento de los contratos por obra y faena (Caro, 2012)- sirven de suficiente evidencia de que formalización y precariedad laboral no se oponen. Otro tanto podría decirse de las formas de contratación que han proliferado entre los trabajadores portuarios, en que los estibadores/desestibadores son frecuentemente contratados a plazo fijo y finiquitados en una misma jornada laboral.

Además de estas salvedades que provienen de la consideración de la aplicabilidad (o no) de la categoría de precariedad en un sentido crossnational, la tesis sobre la precarización vía erosión del estado de bienestar también muestra sus limitaciones si consideramos la larga historia del capitalismo (capitalismo mercantil, capitalismo industrial, capitalismo financiero). Visto así el problema, el período en que dominó el esquema fordista de producción combinado con políticas de promoción del empleo, organización sindical de los trabajadores en democracia, y alguna variante de estado social, no sólo estuvo limitado espacialmente a la experiencia de algunas economías avanzadas - especialmente de Europa, siendo la protección social mucho menor en los Estados Unidos- sino que además temporalmente fue un período más bien corto y excepcional del capitalismo que coincidió con el fordismo y el auge de las políticas keynesianas (Neilson y Rositter, 2008; Ross, 2008).

En definitiva, vemos que mientras algunos analistas sostienen que el incremento de formas atípicas de trabajo en economías avanzadas es el resultado de una paulatina disolución de las formas características de capitalismo regulado y de trabajo estándar propias del régimen fordista (Dörre 2013), otros han criticado esta explicación debido a que supondría la universalización de una particular figura del trabajador -varón, sindicalizado, capacitado, ciudadano- y la consiguiente invisibilización de categorías marginales de trabajadores, entre los que estarían aquellos sin mayor capacitación, las mujeres, migrantes, jóvenes, “discapacitados”, para nombrar sólo algunas categorías de sujeto que no coinciden con la figura dominante de trabajador regular (Huws 2013). En el marco de la discusión del argumento de Standing, lo importante es destacar que éstas categorías de trabajadores precarios no son de reciente aparición y que su presencia no coincide con el retroceso del estado de bienestar, sino que son categorías sociales 
de larga data, tan sólo que se hallaban invisibilizadas por las formas de comprensión dominantes en que hemos heredado de las sociedades avanzadas.

Por otra parte, no es menos cierto que la precariedad, aún cuando se encarna de modo diferente en cada contexto social e histórico, nombra a un proceso general y común que afecta las condiciones de trabajo en todo el mundo. Si bien es fundamental considerar las singularidades de cada contexto, es igualmente importante comprender las características generalizadas de los mecanismos que producen la precariedad laboral, en especial la flexibilización del trabajo, la inseguridad social y la globalización. Debemos avanzar en la comprensión de la articulación de las diferentes fuerzas sociales y económicas que, siendo similares, se presentan diferenciadamente en cada realidad. Por ejemplo, el nivel de debilitamiento de los sindicatos y de movilidad del capital y el trabajo, nunca suceden en un vacío institucional. Las realidades institucionales de cada sociedad facilitan o regulan la influencia de dichas fuerzas sociales y económicas. En sociedades de América Latina el trabajo precario no es sólo una respuesta empresarial a la competencia global por precios, pues en ocasiones los costos laborales están en niveles competitivos por la vía de mantener bajos los ingresos salariales de los trabajadores. En estas sociedades, el trabajo precario frecuentemente ha sido un elemento constitutivo de la estrategia de desarrollo de las élites dirigentes que conciben a sus países como jugadores subalternos sometidos a la influencia de instituciones financieras internacionales. Así, siguiendo los consejos del Fondo Monetario Internacional y del Banco Mundial, varios países del Sur Global han incorporado en sus leyes laborales esquemas de contratación que facilitan la precariedad laboral, y en sus instituciones económicas la desregulación del capital (Lee y Kofman, 2012).

A partir de la lectura de The Precariat, este ensayo distinguió los diferentes mecanismos generalizados que producen la precariedad laboral asociados a los procesos de globalización, inseguridad social y flexibilidad laboral, y propuso que es necesario avanzar en la comprensión de los modos en que dichos mecanismos se articulan entre sí y en que se acoplan con los contextos sociales e históricos más amplios de cada realidad. A pesar de la necesidad de considerar las particularidades de cada realidad, las características generalizadas de los mecanismos que producen la precariedad laboral descritos por Standing (2011 y 2012), en especial la flexibilización del trabajo y la competencia global, nos llevan a sostener que el relato de The Precariat es útil para comprender las nuevas realidades del trabajo en América Latina (y tal vez en todo el Sur Global). Por ello, The Precariat debiera ser considerado por los investigadores y sociólogos del trabajo como una de las piezas fundamentales para discutir y delinear la emergente agenda de investigación sobre la precariedad laboral en América Latina. 


\section{Notas}

${ }^{1}$ Al respecto, remito al lector al "Book Symposium on Guy Standing's The Precariat. The New Dangerous Class" publicado en Work, employment and society 26(4) de 2012. Work, employment and society es una de las principales revistas en la sociología del trabajo.

${ }^{2}$ La expresión sociedad de mercado es de Karl Polanyi. Volveremos sobre ella en un momento.

${ }^{3}$ Es un tema debatido si el precariado es una clase social o, más bien, una categoría social muy heterogénea. El término fue al parecer usado por primera vez por la sociología francesa en los 1980 para aludir a trabajadores temporales (Standing, 2011).

${ }^{4}$ Según Standing, denizen es una categoría característica del Derecho Romano y Medieval que se refería a individuos extranjeros a los que les eran reconocidos algunos derechos. Su traducción más literal al lenguaje actual sería residente legal. Sin embargo, este término no capta el uso metafórico y condensado de Standing, que incluye en esta categoría a una variedad de sujetos nacionales que, no obstante, no son ciudadanos plenos. Cohen (2009) llama semi-ciudadanos a una variedad de categorías de sujeto similares a los denizens de Standing. Ambos coinciden en señalar que la ciudadanía moderna no es completamente inclusiva. 


\section{Bibliografía}

Caro, Pamela (2012), “Magnitud y Características de la participación laboral en el empleo temporal agrícola en Chile”, Revista de Estudios Transfronterizos Si Somos Americanos, Volumen XII, N²2: 51-83.

Cárdenas, Ana et al. (2012), ¿Qué significa el trabajo hoy? Cambios y continuidades en una sociedad global. Catalonia, Santiago de Chile.

Castel, Robert (2009), “Die Wiederkehr der sozialen Unsicherheit”, en Robert Castel y Klaus Dörre (editores) Prekarität, Aufstieg, Ausgrenzung. Die Soziale Frage am Beginn des 21. Jahrhunderts. Campus Verlag, Frankfurt.

Cohen, Elizabeth, F. (2009), Semi-citizenship in Democratic Politics. CUP, Cambridge.

Day, Dorothy (1952), "Poverty and Precarity”, The Catholic Worker, 2, (6). Disponible en: http://www.catholicworker.org/dorothyday/ Reprint2.cfm?TextID=633.

Dörre, Klaus (2009), “La Precariedad: ¿Centro de la cuestión social en el Siglo XXI? Actuel Marx 8: 79-108.

Ídem (2013), presentación en el panel "Concepts and debates aboutprecarization in a German/European perspective”, Workshop "Precarity and Precarization - European and Latin American Perspectives in the Regulation of Labor and Labor Markets”, desigualdades.net, Universidad Libre de Berlin, 25 de Octubre de 2013.

Hall, Stuart (2011), “The Neo-Liberal Revolution”, Cultural Studies Vol. 25, No. 6: 705-728

Hall, Stuart y O’Shea, Alan (2013), “Common-sense Neoliberalism”, Soundings 55, 8-24.

Harvey, David (2007), A Brief History of Neoliberalism. Oxford University Press, Oxford.

Huws, Ursula (2013), presentación en el panel "Concepts and debates aboutprecarization in a German/European perspective”, Workshop "Precarity and Precarization - European and Latin American Perspectives in the Regulation of Labor and Labor Markets”, desigualdades.net, Universidad Libre de Berlin, 25 de Octubre de 2013.

Kalleberg, Arne (2012a), “Job Quality and Precarious Work: Clarifications, Controversies, and Challenges”, Work and Occupations 39 (4): 427-448.

Ídem (2012b), “Globalización y trabajo precario: Desafíos para la sociedad y la sociología”, en Cárdenas, Ana et al., ¿Qué significa el trabajo hoy? Cambios y 
continuidades en una sociedad global. Catalonia, Santiago de Chile.

Lee, Ching Kwan y Kofman, Yelizavetta (2012), “The Politics of Precarity: Views Beyond the United States”, Work and Occupations 39(4) 388-408.

Leiva, Sandra (2012), “Trabajadores autónomos dependientes en Chile y Alemania” en Cárdenas, Ana et al., ¿Qué significa el trabajo hoy? Cambios y continuidades en una sociedad global. Catalonia, Santiago de Chile.

Marshall, Thomas H. (1997), “Ciudadanía y Clase Social”, Reis 79: 297-344

Marx, Karl (2001), El Capital (Volumen I). Siglo XXI, México.

Neilson, Brett y Rossiter, Ned (2008) "Precarity as a Political Concept, or, Fordism as Exception”, Theory, Culture and Society 25 (7-8): 51-72.

Polanyi, Karl (1992), La gran transformación: Los orígenes políticos y económicos de nuestro tiempo. FCE México, México.

Ross, Andrew (2008), "The New Geography of Work. Power to the Precarious?” Theory, Culture and Society 25 (7-8): 31-49.

Sennett, Richard (2000), La Corrosión del Carácter. Anagrama, Barcelona.

Sproll, Martina (2013), "Precarization, Genderization and Neotaylorist Work”, desiguALdades.net Working Paper Series, 44, 1-50.

Standing, Guy (2009), Work After Globalization. Building Occupational Citizenship. Edward Elgar, Cheltenham.

Ídem (2011), The Precariat. The New Dangerous Class. Bloomsbury Academic, Londres y Nueva York.

Ídem (2012), “The Precariat: From Denizens to Citizens?” Polity 44 (4) : 588-608.

Thompson, Edward Palmer (1967), “Time, Work-Discipline and Industrial Capitalism”, Past and Present 38: 56-97.

Ídem (1989), La formación de la clase obrera en Inglaterra, Laia, Barcelona.

Webster, Edward (2005), “Making a Living, Earning a Living: Work and Employment in Southern Africa”, International Political Science Review 25 (1): 55-71. 